\title{
REVIEW
}

\section{Left ventricular free wall rupture: clinical presentation and management}

\author{
J Figueras, J Cortadellas, J Soler-Soler
}

In patients with acute myocardial infarction, left ventricular free wall rupture is an infrequent complication $(2-4 \%)$ but it is associated with a high mortality from pericardial tamponade. ${ }^{1-8}$ It accounts for $5-24 \%$ of all in hospital deaths related to acute myocardial infarction. ${ }^{46910}$ To reduce this high mortality it is important to improve the way in which these patients are classified, as this may help clinicians anticipate myocardial rupture, prevent it occurring, and achieve better therapeutic results when it does occur. In this paper, we provide an update on the clinical, electrocardiographic, echocardiographic, and angiographic features of these patients, identifying the different forms in which free wall rupture presents. We also review the management of these cases and suggest a conservative strategy that may be applied successfully to a selected subgroup of patients.

\section{Clinical profile of patients with free wall rupture}

The clinical characteristics of patients with free wall rupture are summarised in table 1 . Characteristically, free wall rupture occurs in relatively elderly patients, generally older than 55 years and usually between 65 and $70,{ }^{13-5} 11-13$ without any apparent sex bias $^{314-17}$-although it may be relatively more common in female patients in view of the lower incidence of acute myocardial infarction in women. ${ }^{45}$ In most cases the myocardial infarct is the first one recorded and it is usually transmural ${ }^{2}{ }^{12-14} 18$ but without overt heart failure. $^{2131418}$ There is an infrequent history of angina pectoris. ${ }^{21314} 19$ The incidence of diabetes is not particularly high, though arterial hypertension is common-often more than $50 \%$, but with some exceptions. ${ }^{156121418}$ These patients present with a rather prolonged episode of anginal pain, often lasting for four to six hours or more, ${ }^{124515162021}$ but sometimes with pain of shorter duration that has been preceded by other episodes lasting more than 30-60 minutes in the days leading up to the precipitating event. ${ }^{16}$ Not infrequently these patients experience delay in admission to hospital, ${ }^{291618}$ often because of misdiagnosis but occasionally because of a silent acute myocardial infarct complicated by pericardial tamponade and manifested as syncope or systemic hypotension. This delay in hospital admission
Table 1 Clinical characteristics of patients with free wall rupture

Age $>55$ years (67 average)

First transmural myocardial infarction

Killip class I or II

Persistent ST segment elevation

Persistent or recurrent chest pain

Sudden or progressive hypotension or sudden

electromechanical dissociation

is often associated with maintenance of some ambulatory activities. ${ }^{216}{ }^{18}$ Additional risk factors, or triggering factors, for free wall rupture are the presence of persistent arterial hypertension ( $\geqslant 150 \mathrm{~mm} \mathrm{Hg}$ ) during the first 10-24 hours of the acute infarction while in hospital at rest, and any undue physical effort such as persistent coughing, vomiting, or agitation (table 2). ${ }^{291516182022}$

On admission, the ECG is consistent with transmural acute myocardial infarction, with a notably elevated ST segment which tends to remain persistently elevated over the ensuing hours or days. ${ }^{213} 15$ Thus patients with pronounced ST segment elevation and sustained arterial hypertension are particularly likely to have an early free wall rupture. ${ }^{13}$ Nevertheless, patients who are otherwise at risk of ventricular rupture but who present with an electrocardiographically "small" lateral infarct, with little ST segment elevation, and occasionally with small Q waves, and in whom control of arterial hypertension may not appear so important, ${ }^{13}$ also deserve special attention. Although free wall rupture occurs in infarcts of variable size, it is not infrequently found in association with a rather small area of lateral necrosis. ${ }^{12} 182^{23-25} \mathrm{In}$ some cases there may be associated left ventricular hypertrophy, and this seems to increase the risk of free wall rupture. ${ }^{1} 214$ In-hospital free wall rupture is often preceded by chest pain similar to that of acute myocardial infarction ${ }^{12} 51516202326$; this may be associated with re-elevation of the ST segment or with flat or negative $\mathrm{T}$ waves becoming positive. $^{21327}$

Table 2 Factors facilitating free wall rupture

Delayed hospital admission ( $>12-24$ hours)

Persistent systemic hypertension during first $>10-24$ hours

"Unusual" in-hospital physical effort (agitation, repetitive

vomiting or coughing, etc)

Extension of myocardial infarction

Expansion of myocardial infarction 
The significance of the pain and the ECG changes is unclear and they may not always have the same cause. Extension of the infarct could be an explanation in some cases, particularly those with late rupture, ${ }^{116182829}$ and necropsy studies have confirmed this histologically. ${ }^{16}{ }^{18}{ }^{28}$ Rupture of the necrotic tissue could also be a mechanism, ${ }^{2} 3031$ as could the entrance of blood into the pericardial cavity. ${ }^{15}$ In some cases, the chest pain and ECG changes with hypotension may simply indicate extension of the infarct without free wall rupture. ${ }^{32}{ }^{33}$ However, when hypotension develops within a few minutes after the onset of pain, and particularly when it is rapidly followed by electromechanical dissociation, ${ }^{2} 34$ the possibility of a free wall rupture is higher than when it develops over a longer period of time. Definite diagnosis of free wall rupture would, however, require evidence of pericardial effusion or tamponade on echocardiography.

\section{Clinical presentation}

The types of free wall rupture according to time of occurrence or form of presentation are outlined in table 3. Depending on the time of its occurrence, the rupture may be early, when it develops within the first 48 hours, or late, when it develops beyond the second day. The early form represents $40-50 \%$ of cases, ${ }^{15} 131435$ but the true proportion is likely to be higher because it will also include patients dying suddenly from free wall rupture before reaching hospital. ${ }^{1136}$ Strain in the infarcted zone because of sustained arterial hypertension $^{2} 1213182337$ or the maintenance of ambulatory activity ${ }^{16} 182022$ may favour this complication. In patients with early rupture there is hardly any thinning of the infarcted area, whereas late rupture generally develops in an already expanded infarcted tissue. ${ }^{1324} 25$ Late rupture also appears to be less affected by arterial hypertension, but undue physical exercise-such as persistent vomiting, coughing, or excessive effort when using a bedpan ${ }^{216}{ }^{18}$ - can often be the underlying trigger. In some instances, infarct extension may also be a trigger, by making an infarct fully transmural. ${ }^{18} 28$

According to the type of presentation, free wall rupture may also be considered acute or subacute. There is ample agreement that the

Table 3 Types of free wall rupture according to time of occurrence or form of presentation

\begin{tabular}{|c|c|}
\hline \multirow{2}{*}{\multicolumn{2}{|c|}{ Time of occurrence }} \\
\hline & \\
\hline 1. Early rupture ( $\leqslant 48$ hours) & $\begin{array}{l}\text { - delayed hospital admission } \\
\text {-persistent pain (>4-6 hours) } \\
\text {-acute arterial hypertension } \\
\text {-frank and persistent ST segment elevation (except in lateral } \\
\text { infarctions) }\end{array}$ \\
\hline 2. Late rupture ( $>48$ hours) & $\begin{array}{l}\text {-recurrent chest pain } \\
\text {-persistent ST segment elevation } \\
\text {-"undue" physical exercise } \\
\text {-infarct extension } \\
\text {-infarct expansion }\end{array}$ \\
\hline \multicolumn{2}{|l|}{ Form of presentation } \\
\hline 1. Acute rupture & $\begin{array}{l}\text {-acute tamponade with sudden electromechanical } \\
\text { dissociation or severe hypotension }\end{array}$ \\
\hline 2. Subacute rupture & $\begin{array}{l}\text {-moderate to severe pericardial effusion: } \\
\text { (a) with tamponade and haemodynamic compromise } \\
\text { with modest or progressive hypotension } \\
\text { (b) without tamponade }\end{array}$ \\
\hline
\end{tabular}

acute form is associated with sudden death caused by severe hypotension and electromechanical dissociation secondary to acute pericardial tamponade..$^{3-51315}$ This is accompanied by cyanosis of the neck and face and intense jugular venous congestion. The definition of the subacute form-a term coined by $\mathrm{O}^{\prime}$ Rourke $^{38}$ some years ago-is less clear. The term was originally applied to cases where there was acute tamponade associated with severe hypotension or electromechanical dissociation which could be temporarily reversed by resuscitative manoeuvres, allowing subsequent surgical repair. ${ }^{38}{ }^{39}$ At present, however, there is no clear distinction between "acute" and "subacute" rupture when associated with electromechanical dissociation or severe hypotension, other than that the former is fatal while the latter is compatible with survival beyond the initial medical management. In order to make a more satisfactory distinction between these two terms, we propose that the term "subacute free wall rupture" be reserved for cases with moderate to severe pericardial effusion and hypotension of varied severity, usually associated with sinus bradycardia or nodal rhythm and jugular venous congestion, whereas the term "acute" should be limited to those causing cardiac arrest by electromechanical dissociation, either of sudden onset or following severe hypotension. The implications of this new definition are that, although both presentations may be successfully managed by medical treatment initially, the subacute form is clearly less severe and hence more compatible with survival. In addition, the new definition would allow the incorporation of subacute forms of different severity, ranging from severe hypotension and syncope to negligible haemodynamic compromise..$^{39} 40$

The subacute form has been recognised increasingly and may account for up to $30 \%$ of all cases of in-hospital free wall rupture. ${ }^{15920212638-41}$ However, the existence of a subset of patients with "mild" subacute free wall rupture has received little attention. ${ }^{39}$ These patients, who meet the basic free wall rupture clinical profile - that is, age $>55$ years, first transmural acute myocardial infarct, persistent ST segment elevation, and without overt heart failure - present with a moderate to severe pericardial effusion but without haemodynamic compromise. The effusion is usually identified on a routine postinfarction echocardiogram. In our experience, this condition should alert the clinician to the possibility of a "silent" subacute free wall rupture, as we have witnessed several cases where such a possibility was not considered but where the patient subsequently died of a re-rupture with tamponade, confirmed at necropsy. Hypothetically, however, some of these cases of haemopericardium, particularly those linked to mild forms of subacute free wall rupture, could be attributed to others causes such as a haemorrhagic infarction or a haemorrhagic pericarditis. ${ }^{26} 384243$ Necropsy documentation of these kinds of haemopericardium is, however, rather rare, ${ }^{42}{ }^{43}$ and existing reports refer to cases with or without tamponade that have been operated on and 
in whom no apparent free wall rupture was observed..$^{26} 38$ Notwithstanding these interpretations, ${ }^{26}{ }^{41-43}$ we believe that in a proportion of these surgical cases the possibility that a healed rupture had been overlooked should have been considered. There are indeed some necropsy studies of acute myocardial infarction with haemopericardium in which a free wall rupture that was not apparent macroscopically ${ }^{413} 18$ corresponded histologically to a thrombosed myocardial dissection. ${ }^{41}{ }^{44}$ Moreover, some of these operated cases, apparently unrelated to a free wall rupture, later developed a left ventricular pseudoaneurysm. ${ }^{45} 46$

It is therefore our contention that some self limited free wall ruptures are rather small and are probably only open temporarily during bursts of arterial hypertension or increased myocardial strain. Some may even just represent stuttering oozing through a narrow fissure. ${ }^{17}{ }^{41}$ The subsequent organisation of the haematoma would lead to an initial healing by fibrin deposits that would progressively evolve into definite healing by pericardial adhesions.

\section{Management}

The management of free wall rupture is outlined in table 4 . Although this complication, with its various presentations, is by far the most common cause of haemopericardium during acute myocardial infarction, especially when recognised risk factors for this complication are present, a definitive clinical diagnosis of free wall rupture can only be obtained at thoracotomy, though with some limitations, ${ }^{41}{ }^{44-46}$ or occasionally by the echocardiographic ${ }^{40} 47$ or angiographic ${ }^{162647-49}$ demonstration of blood leaking into the pericardial cavity. This is the reason why it is the management of haemopericardium that is discussed rather than of free wall rupture. We shall distinguish between haemopericardium with and without haemodynamic compromise.

\section{HAEMOPERICARDIUM WITH HAEMODYNAMIC COMPROMISE \\ Initial treatment}

Patients who fulfil the clinical profile of risk for free wall rupture and who experience sudden non-arrhythmic hypotension unrelated to the administration of $\beta$ blockers or vasodilators, but associated with sinus bradycardia or nodal rhythm and jugular distension, should be assumed to have acute tamponade. They

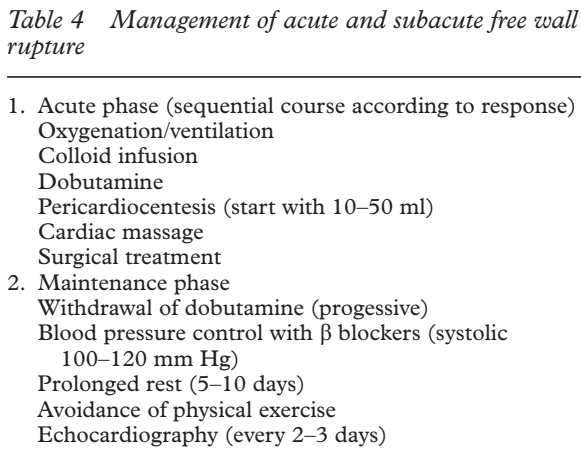

should immediately be given fluid replacement with colloidal solutions and dobutamine at $5-10 \mu \mathrm{g} / \mathrm{kg} / \mathrm{min}$, and an echocardiogram should be performed as soon as possible to assess the presence of a pericardial effusion, with or without signs of right atrial compression. If haemodynamic recovery is readily achieved, a maintenance protocol (see below) will be followed and the cardiac surgical team informed of the likely need for intervention if haemodynamic stabilisation is not maintained. If no cardiac surgery facilities are available the patient should be transferred to another centre with these facilities. If the patient remains hypotensive with signs of peripheral hypoperfusion, pericardiocentesis should be carried out and a small quantity of pericardial fluid $(10-50 \mathrm{ml})$ withdrawn-sufficient to achieve haemodynamic recovery and an adequate urine output. A second pericardiocentesis can be performed if recovery is partial or absent, or if tamponade recurs. If, following pericardiocentesis, recovery is achieved and sustained, the maintenance protocol may be followed, but if recovery is unsuccessful after two pericardiocenteses, an emergent thoracotomy should be undertaken. The optimal surgical treatment is the application of a Teflon ${ }^{39} 50$ or pericardial patch $^{51}$ to the epicardial surface of the ruptured site with cyanoacrylate glue, ${ }^{50}$ preferably without the use of cardiopulmonary bypass. This may be difficult if active bleeding is present after opening the pericardium. In this case cardiopulmonary bypass may facilitate patch application. Infarctectomy of the rupture zone followed by a Teflon buttressed suture ${ }^{52}$ should, on the other hand, be limited to cases where the use of a patch is unsuccessful, because it may cause excessive reduction in the size of the ventricular cavity.

When tamponade is complicated by cardiac arrest because of electromechanical dissociation, cardiac massage may be necessary to restore an adequate pulse, in addition to the measures outlined above. In these instances, endotracheal intubation and mechanical ventilation will also be necessary, and a pericardiocentesis may be required before an echocardiogram can be performed. If there is no haemodynamic recovery in spite of all these measures, which may also include the administration of adrenaline (epinephrine), emergency surgical treatment should be attempted, as this may be lifesaving in some instances. ${ }^{1626394152}$ Where recovery is slow, particularly in patients with large infarcts, it may be necessary to monitor the right and left ventricular filling pressures through a Swan-Ganz catheter to assess the haemodynamic response to treatment and the possible reappearance of tamponade.

\section{Maintenance treatment}

Once haemodynamic recovery has been achieved, a conservative approach may be followed. This includes maintenance of blood pressure between 100 and $120 \mathrm{~mm} \mathrm{Hg}$ to avoid undue myocardial wall strain but to preserve urine output. In some patients with large acute myocardial infarcts, where there is often 
myocardial hypocontractility, the use of dobutamine with or without frusemide (furosemide) may still be necessary for several hours or days to maintain an adequate urine output in the absence of tamponade. As soon as possible, however, inotropic support should be withdrawn and replaced by $\beta$ blocking agents as tolerated, initially propranolol because it is easier to titrate than other $\beta$ blockers, to reduce myocardial workload as much as possible. To further reduce cardiac strain, bed rest should be extended to 4-8 days and any undue physical effort-such as excessive straining during the use of a commode or other isometric exercise, repetitive vomiting or coughing-should be avoided. A routine postinfarction discharge exercise stress test should be postponed for at least two months. Anticoagulant treatment should be withheld but antiaggregant treatment with low dose aspirin may probably be continued.

The patient should be nursed in the coronary care unit for the first 7-10 days, depending upon the severity of the initial hypotension, the presence of electromechanical dissociation, the magnitude of the pericardial effusion, overall haemodynamic status, and the difficulty in controlling the blood pressure. An echocardiogram should be repeated every two to four days, depending on the haemodynamic course, to assess changes in the pericardial effusion. If the patient has a second episode of tamponade but arterial hypotension is not severe, a repeat pericardiocentesis may be performed and if the result is satisfactory maintenance treatment may be resumed. This option would be particularly appropriate for patients at high risk of surgery because of old age, comorbidity (severe diabetes, peripheral vascular disease, advanced renal or chronic pulmonary disease, previous cerebrovascular accident), a large infarct, especially one with posterior localisation, and so on. On the other hand, if these relative contraindications are not present and if the recurrence of tamponade is severe or not readily reversed, surgical treatment is indicated following repeat pericardiocentesis. Pericardial fluid aspiration should again be limited to $10-40 \mathrm{ml}$, the aim being to stabilise the patient's haemodynamic state before surgery.

\section{Early follow up treatment}

During the first two months after hospital discharge, the patient should be allowed to walk but should be advised to avoid any relatively strenuous exercise, particularly isometric stress. Blood pressure should be strictly controlled ( $\leqslant 120 / 80 \mathrm{~mm} \mathrm{Hg}$ ) and $\beta$ blockers given as tolerated. Echocardiography is advisable at one month to assess the pericardial effusion.

\section{HAEMOPERICARDIUM WITHOUT HAEMODYNAMIC} COMPROMISE

From our own experience, patients with haemopericardium but no haemodynamic compromise can receive the maintenance treatment and early follow up treatment outlined above. A diagnostic pericardiocentesis may be useful to confirm the existence of a haemopericardium.

\section{Previous experience of a conservative approach to free wall rupture/ haemopericardium}

Between January 1986 and April 1999 we carried out a prospective evaluation of the conservative management of patients with acute myocardial infarction and clinically suspected free wall rupture, incorporating an alternative surgical approach when medical treatment failed. During this period we managed 141 patients with a suspected free wall rupture-120 with acute haemodynamic compromise and 21 without. Of the former, $86 \mathrm{did}$ not achieve haemodynamic recovery during resuscitative manoeuvres: 17 of these were operated on within the first three hours, with two long term survivors, whereas 69 could not be operated on and died shortly afterwards. The remaining 55 patients responded to the initial medical treatment and underwent conservative management. Four of these, however, were operated on within the following 48 hours because of recurrence of tamponade despite pericardiocentesis. In three, a large Teflon patch was glued to the epicardium with cyanoacrylate over the rupture site; in the fourth, a sealed free wall rupture with haemopericardium was found and was not repaired. Of the 51 patients who did not have surgery, 30 had presented with tamponade and 21 had not. In five of the patients with tamponade, however, and in agreement with the surgical team, surgery was not undertaken because the operative risk was considered too high.

The mean (SD) width of the pericardial effusion in patients with haemodynamic compromise was $22.1(4.7) \mathrm{mm}$, and in those without compromise, $16.3(5.7) \mathrm{mm}$. Independent of haemodynamic compromise, most patients showed fibrin deposits in the pericardial space, generally near the apex. A pericardiocentesis was performed in 31 patients: haemopericardium was confirmed in 30 and a serosanguineous effusion was present in one. In 26 patients, a contrast left ventriculogram was performed; in six of these a leak of contrast material into the pericardial space was observed, while in another, blood flow from the left ventricle to the pericardium was detected by Doppler echocardiography.

Of the 51 patients who did not have surgical treatment, 16 died during their hospital stay. Ten patients died suddenly following rerupture and tamponade, confirmed at necropsy in seven and by echocardiography in three, while six died of other causes - two of sepsis, two of respiratory distress, and two of myocardial hypocontractility. In three of these latter six patients, a necropsy was performed and a sealed free wall rupture was documented. In five of the 10 patients with re-rupture, surgical treatment had been excluded because of excessive risk, and in another, surgery was not considered because the effusion was moderate (only $10 \mathrm{~mm}$ ) and there was no haemodynamic compromise. Three of the five patients without 
surgical contraindications were among the first eight patients included in the study.

During the follow up of the 36 patients who were eventually discharged, sequential echocardiography did not show the development of a pseudoaneurysm. Five patients died, two of gastric cancer, at three months and 25 months, one of progressive heart failure without pericardial effusion at 25 months, one of a cerebrovascular accident at eight months, and another of chronic lung disease at 18 months. Four of the remaining 31 patients underwent cardiac surgery at $32,43,63$, and 321 days after free wall rupture, two for coronary bypass, one for a concomitant small septal rupture, and one cardiac transplant. A sealed free wall rupture was observed and was deemed to be repaired in one patient. The mean (SD) follow up for the 29 survivors in whom the free wall rupture was not repaired was of 72 (31) months.

This is the first prospective series involving a conservative approach to ventricular free wall rupture, and it includes some previously reported cases. ${ }^{40}$ However, there have also been isolated reports of other cases surviving without surgery. ${ }^{8} 475354$ Thus some patients in whom there is a strong suspicion of free wall rupture may be successfully managed without operation, although in many cases surgical treatment is still the only alternative.

\section{Summary}

Diagnosis of free wall rupture is strongly suspected when a patient with a high risk profile-age $>55$ years, first transmural acute myocardial infarct, absence of overt heart failure, persistent ST segment elevation, prolonged pain during the acute phase-presents with sudden hypotension or electromechanical dissociation, often preceded by recurrence of chest pain, together with raised jugular venous pressure and a moderate to severe pericardial effusion on echocardiography. To avoid semantic confusion, it is proposed that the term "acute free wall rupture" be reserved for patients with cardiac arrest caused by electromechanical dissociation or severe hypotension, and "subacute" rupture be applied to patients with hypotension of lesser degree.

When cardiac arrest has occurred, management includes cardiac massage, ventilatory support, administration of inotropic agents and colloids, and pericardiocentesis. If improvement occurs, conservative management is then continued in close consultation with the surgical team. If initial management does not result in improvement, emergency thoracotomy is performed, preferably without cardiopulmonary bypass, and the rupture site is repaired with a Teflon patch glued to the epicardium. When the manifestation of free wall rupture is hypotension rather than cardiac arrest, initial management should involve colloid infusion, dobutamine infusion, and pericardiocentesis with aspiration of sufficient pericardial fluid $(10-50 \mathrm{ml})$ to restore haemodynamic competence and urine output. Conservative management is then continued. If haemodynamic recovery cannot be achieved, surgical treat- ment is indicated. In all cases, conservative management involves blood pressure control $(\beta$ blocker treatment) and avoidance of physical stress (bed rest for 5-7 days).

A conservative approach is particularly indicated in patients with important comorbidity factors that greatly increase the surgical risk, such as severe chronic lung disease, renal failure, extensive myocardial infarction, or serious peripheral vascular disease. This method of treatment, which represents a challenge in most settings and contravenes the standard surgical approach in all cases of suspected free wall rupture, will need to be validated by a substantial cohort of patients from more than one institution.

1 London RE, London SB. Rupture of the heart. A critical nalysis of 47 consecutive autopsy cases. Circulation 1965;31:202-8

2 Friedman HS, Kuhn LA, Katz AM. Clinical and electrocardiographic features of cardiac rupture following acute myocardial infarction. Am f Med 1971;50:709-20.

3 Bates R, Beutler S, Resnekov L, et al. Cardiac rupturechallenge in diagnosis and management. Am $\mathcal{f}$ Cardiol 1977;40:429-37.

4 Rasmussen S, Leth A, Kjoller E, et al. Cardiac rupture in acute myocardial infarction. A review of 72 consecutive cases. Acta Med Scand 1979;205:11-16.

5 Dellborg M, Held P, Swedberg K, et al. Rupture of the myocardium. Occurrence and risk factors. Br Heart $\mathcal{F}$ 1985;54: 11-16.

6 Shapira I, Isakow A, Burke M, et al. Cardiac rupture in patients with acute myocardial infarction. Chest 1987;92: $219-23$

7 Pollack H, Miczoch J. Effect of nitrates on the frequency of left ventricular free wall rupture complicating acute myocardial infarction: a case-controlled study. Am Heart $\mathcal{F}$ 994;128:446-71

8 Blinc A, Noc M, Pohar B, et al. Subacute rupture of the left ventricular free wall after acute myocardial infarction. Three cases of long-term survival without emergency surgery. Chest 1996;109:565-7.

9 Lewis AJ, Burchell HB, Titus JL. Clinical and pathologic features of post-infarction cardiac rupture. Am $\mathcal{f}$ Cardiol 1969;23:43-53.

10 Reddy SG, Roberts WC. Frequency of rupture of the left ventricular free wall or ventricular septum among necropsy
cases of fatal acute myocardial infarction since introduction of coronary care units. Am F Cardiol 1989;63:906-11.

11 Batts K, Ackermann DM, Edwards WD. Postinfarction rupture of the left ventricular free wall: clinicopathologic correlates in 100 consecutive autopsy cases. Hum Pathol 1990;21:530-5.

12 Naeim F, De la Maza LM, Robbins SL. Cardiac rupture during myocardial infarction. A review of 44 cases. Circulation 1972;45:1231-9.

13 Figueras J, Curos A, Cortadellas J, et al. Relevance of electrocardiographic findings, heart failure, and infarct site in assessing risk and timing of left ventricular free wall rupture during acute myocardial infarction. Am $\mathrm{f}$ Cardiol 1995;76:543-7.

4 Mann JM, Roberts WC. Rupture of the left ventricular free wall during acute myocardial infarction: analysis of 138 necropsy patients and comparison with 50 necropsy patients with acute myocardial infarctions without rupture. Am F Cardiol 1988;62:847-59.

15 Oliva PO, Hammill SC, Edwards WE. Cardiac rupture, a clinically predictable complication of acute myocardial infarction: report of 70 cases with clinicopathologic correlations. F Am Coll Cardiol 1993;22:720-6.

16 Figueras J, Cortadellas J, Calvo F, et al. Relevance of delayed hospital admission on development of cardiac rupture during acute myocardial infarction. Study in 225 patients with free wall, septal or papillary muscle rupture. 7 Am Coll Carfree wall, septal or papil

17 Purcaro A, Constantini C, Ciampani N, et al. Diagnostic criteria and management of subacute ventricular free wall rupture complicating acute myocardial infarction. $A m \mathcal{F}$ Cardiol 1997;80:397-405.

18 Wessler S, Zoll PM, Schlesinger MJ. The pathogenesis of spontaneous cardiac rupture. Circulation 1952;6:334-51.

19 Nakano M, Konishi T, Takezawa H. Potential prevention of myocardial rupture resulting from acute myocardial infarction. Clin Cardiol 1985;8:199-204.

20 Lautsch FV, Lanks KW. Pathogenesis of cardiac rupture. Arch Pathol 1967;84:264-71.

21 Feneley MP, Chang VP, O'Rourke MF. Myocardial rupture after acute myocardial infarction. Ten year review. Br Heart f 1983;49.550-6.

22 Jetter WW, White PD. Rupture of the heart in patients in mental institution. Ann Intern Med 1944;21:783-94.

23 Edmonson HA, Hoxie HJ. Hypertension and cardiac rupture. A clinical and pathologic study of seventy-two cases in thirteen of which rupture of the interventricular cases in thirteen of which rupture of the interve
septum occurred. Am Heart f 1942;24:719-33. 
24 Schuster EH, Bulkley BH. Expansion of transmural myocardial infarction: pathophysiologic factor in cardiac myocardial infarction: a pathophysio
rupture. Circulation 1979;60:1532-8.

25 Saffitz JE, Fredrickson RC, Roberts WC. Relation of size of transmural acute myocardial infarct to mode of death, interval between infarction and death and frequency of coronary arterial thrombi. Am f Cardiol 1986;57:1249-54

26 Windsor HM, Chang VP, Shanahan MX. Postinfarction cardiac rupture. $\mathcal{F}$ Thorac Cardiovasc Surg 1982;84:755-61. 27 Mir MA. Prognostic value of an electrocardiographic sign in acute myocardial infarction. Am Heart f 1972;84:182-8.

28 Silvestri F, Stanta G, Constantinides F, et al. La rottura di cuore come complicanza dell'infarto acuto del miocardio. Considerazioni anatomoistopatologiche sulla base di 15 osservazionei autopiche. Pathologica 1980;72:491-500.

29 Nakamura F, Minamino T, Higashino Y, et al. Cardiac free wall rupture in acute myocardial infarction: ameliorative effect of coronary reperfusion. Clin Cardiol 1992;15:24450 .

30 Datta BN, Bowes VF, Silver MD. Incomplete rupture of the heart with diverticulum formation. Pathology 1975;7:17985.

31 Freeman WJ. The histologic patterns of ruptured myocardial infarcts. Arch Pathol 1958;65:646-53.

32 Figueras J, Cinca J, Valle V, et al. Prognostic implications of early spontaneous angina after acute transmural myocardial infarction. Int $\mathcal{F}$ Cardiol 1983;4:261-72.

33 Bosch X, Theroux P, Waters DD, et al. Early postinfarction ischemia: clinical, angiographic and prognostic significance. Circulation 1987;75:988-95.

34 Figueras J, Curós A, Cortadellas J, et al. Reliability of electromechanical dissociation in the diagnosis of left ventricular free wall rupture in patients with acute myocardial infarction. Am Heart f 1996;131:861-4.

35 Van Tassel RA, Edwards JE. Rupture of heart complicating myocardial infarction. Analysis of 40 cases includir . examples

36 Shirani J, Berezowski K, Roberts WC. Out-of-hospital sudden death from left ventricular free wall rupture during acute myocardial infarction as the first and only manifestaacute myocardial infarction as the first and only manifestation of atherosclerotic

37 Christensen D, Fond M, Reading J, et al. Effect of hypertension on myocardial rupture after acute myocardial infarction. Chest 1977;72:618-22.

38 O'Rourke MF. Subacute heart rupture following myocardial infarction: clinical features of a correctable condition. Lancet 1973;ii: $124-6$.

39 Lopez-Sendon J, Gonzalez A, Lopez de Sa E, et al. Diagnosis of subacute ventricular wall rupture after acute myocardial infarction: sensitivity and specificity of clinical, hemodynamic and echocardiographic criteria. $f$ Am Coll Cardiol 1992;19:1145-53.
40 Figueras J, Cortadellas J, Evangelista A, et al. Medical management of selected patients of left venrticular free wall upture during acute myocardial infarction. $f \mathrm{Am}$ Coll Cardiol 1997;29:512-18.

41 Balakumaran K, Verbaan CJ, Essed CE, et al. Ventricular free wall rupture:sudden, subacute, slow, sealed and stabilized varieties. Eur Heart $\mathcal{F}$ 1984;5:282-8.

42 Lange HF, Aarseth S. The influence of anticoagulant therapy on the occurrence of cardiac rupture and hemopericardium following heart infarction. II. A controlled study of a selected treated group based on 1044 autopsies. Am Heart F 1958;56:257-63.

43 Barbour HB, Hirst AE, Johns VJ. Nontraumatic hemopericardium. An analysis of 105 cases. Am $\mathcal{F}$ Cardiol $1961 ; 8: 102-8$.

44 Torp-Pedersen C, Hansen FS, Pedersen A. Relation of left ventricular free wall rupture in acute myocardial infarction to forced immobilization. Am $\mathcal{7}$ Cardiol 1988;61:910-12.

45 Shabbo FP, Dymond DS, Rees GM, et al. Surgical treatment of false aneurysm of the left ventricle after myocardial infarction. Thorax 1983;38:25-30.

46 Kolibash AJ, Magorien RD, Bush CA, et al. Long-term survival following cardiac rupture with subsequent development of left ventricular pseudoaneurysm. Cathet Cardiovasc Diagn 1982;8:409-17.

47 Raitt MH, Kraft CD, Gardner CJ, et al. Subacute ventricular free wall rupture complicating myocardial infarction. Am Heart f 1993;126:946-55.

48 John LCH, O'Riordan JB. Peri-infarct pursestring for repair of subacute cardiac rupture. Ann Thorac Surg 1996;61: 728-30.

49 Grollier G, Seanu P, Babatasi G, et al. Rupture subaiguës de la paroi libre du coeur. Aspects cliniques, échocardiographiques et anatomiques à propos de $10 \mathrm{cas}$. Arch Mal Graphiques et anatomiques

50 Padró JM, Mesa JM, Silvestre J, et al. Subacute cardiac rupture: repair with a sutureless technique. Ann Thorac Surg 1993;55:20-4.

51 Almdahl SM, Hotvedt R, Larsen U, et al. Postinfarction rupture of left ventricular free wall repaired with a glued-on pericardial patch Case report. Scand 7 Thorac Cardiovasc Surg 1993;27:105-7.

52 Cobbs BW, Hatcher CR, Robinson $\mathrm{PH}$. Cardiac rupture: two long-term survivals. FAMA 1973;223:532-5.

53 Griffith GC, Hegde B, Oblath RW. Factors in myocardial rupture. An analysis of two hundred and four cases at Los Angeles County Hospital between 1924 and 1959. Am F Cardiol 1961;8:792-8.

54 Vasilomanolakis EC, Famularo MA, Kozlowski J, et al. Cardiac rupture following intracoronary streptokinase. Catheter Cardiovasc Diagn 1983;9:291-6. 